\title{
A FRIENDLY OVERVIEW OF NONCOMMUTATIVE GEOMETRY*
}

\author{
ANDRZEJ SitARZ ${ }^{\dagger}$ \\ The Marian Smoluchowski Institute of Physics, Jagiellonian University \\ Reymonta 4, 30-059 Kraków, Poland
}

(Received November 20, 2013)

\begin{abstract}
We present a brief overview of tools and methods of noncommutative geometry and its applications to theoretical physics. Starting with some mathematical background and basic foundations of NCG, we describe the new notion of geometry and demonstrate that changing the paradigm of geometry allows to interpret the physical reality of fundamental interactions from a completely new angle. We present some easy do-it-yourself toy models, which help to understand the principles and results hidden behind the interpretation of the Standard Model in the language of noncommutative geometry.
\end{abstract}

DOI:10.5506/APhysPolB.44.2643

PACS numbers: 02.40.Gh

\section{Introduction}

The current status of fundamental physical theories appears to be only partially satisfactory. On the one hand, the description of basic interactions of particles is evidently a great success - with the recent discovery [1] of the Higgs boson, all pieces of the Standard Model are put together and give a working and correct description of the elementary bricks of the matter. On the other hand, the arguments that the universe is filled with dark matter and dark energy [3] prove that general relativity holds on a large scale in space and was valid even at the early time of the universe history. Yet the origin of both dark matter and dark energy remains a mystery as well as the penultimate quest of physicists: a dream to unify Quantum Field Theory and gravity.

* Based on talks presented at the LIII Cracow School of Theoretical Physics "Conformal Symmetry and Perspectives in Quantum and Mathematical Gravity", Zakopane, Poland, June 28-July 7, 2013.

$\dagger$ Supported by the grant from The John Templeton Foundation. 
That Holy Grail of theoretical physics, despite various attempts and huge efforts is still unattainable thus raising the questions about the validity of our understanding of the world. Neither the String Theory (and its various modifications and spin-offs) nor the Loop Quantum Gravity, which are often presented as the two best candidates for the solution of this puzzle offer the answer. In fact, none of the currently studied models gives an answer that would be even remotely close to the solution.

If everything fails, then one has to start thinking why - and one of the possible answers is that to go this one step forward we need to break the paradigms of contemporary physics. The first one is that the world is geometry and it is justified by the purely geometric description of gravity through Riemannian (or, more correctly: pseudo-Riemannian) geometry and the Yang-Mills theory describing all of gauge theories responsible for fundamental particle interactions. The second paradigm is quantisation, which describes the spectra of atoms, together Quantum Field Theory which describes (albeit in a restricted way) many fine effects of particle interactions. It is remarkable that the both paradigms are separate and there is no clear and well-understood passage from the classical geometric world into the quantum world. Therefore, if we fail to use these tools separately, maybe the necessary step is to look for a more general mathematical theory, which would imply both geometry as well a quantum theory. Noncommutative geometry is a proposition, which goes into this direction.

\section{Why noncommutative geometry?}

Before we start describing what is this theory, let us focus on some mathematical and physical motivations. Geometry is based on the principle of describing spaces, which are sets of points equipped with an additional structure. Yet, physically a point is an elusive theoretical concept and, moreover, even though we describe the world as a space (a collections of points), effectively, we always use coordinates, which are functions on the space. Therefore, the notion of a function (in particular a continuous function) appears to be more fundamental than that of a point. In physics, this is clearly visible when we take into account quantum effects, in particular, the Heisenberg uncertainty principle. Then, it is impossible (both in theory and in practice) to observe a point or to fix the coordinates with an arbitrarily small accuracy.

Even more important is, however, that the classical phase space (space of possible positions and momenta) of a physical object is no longer a space in quantum theory. Instead of using points, we already know that what we see as coordinates are, in reality, operators on a Hilbert space. Moreover, what we usually describe as a state of a physical object (by giving the coordinates 
describing position and momenta) corresponds to the expectations values of these operators for a given state (a normalized vector) in the Hilbert space.

This change of paradigm, which happened around 100 years ago, is quite astonishing, yet in the development of physics that followed there were very few attempts to go forward and abandon the notion of space altogether. Noncommutative geometry is the first sound mathematical concept, which offers the consistent way of creating a geometry of quantum-like counterparts of spaces - algebras.

Noncommutative geometry is not a finished chapter yet, it is a vast theory, which is being developed, which has a lot of mathematical applications (starting from the theory of $C^{*}$-algebras, $K$-theory, index theorem, Hopf algebras etc.) as well as many interesting physical ones (the Standard Model, Quantum Hall Effect, quantum computing, effective quantum field theories, integrable systems).

The aim of this exposition is to demonstrate that noncommutative geometry provides tools, which could be effectively used to construct physical models. Then, in turn, one can invert the problem and by looking at the world (in particular at the particle physics, described by the Standard Model) try to recover the geometry behind that reality - expressed in noncommutative terms.

The long term goal is certainly to provide a meaningful definition of geometry, which would allow to incorporate both the fundamental interactions as we know them with the notion of quantised space (for some arguments and models, see [19]).

\section{From spaces to algebras}

As we noted in the introduction, we do not actually see points of a space. Of course, we can easily visualise a space, in particular, finite and discrete spaces (lattices) as a collection of what we call points, however, to describe any continuous space or manifold we need coordinates. A good notion of a space is, therefore, provided by a collection of well-chosen functions, which we then "measure" to fix an event or a place. Since we know that the world distinguishes between places or events that are near or far from each other, we necessarily introduce a very rough notion of what it means to be close to each other. In the language of spaces, it is topology (this helps to distinguish points) which then is translated to continuous functions. A good set of coordinates on a space is, therefore, a collection of continuous functions, which separate the points. Then, using it to build all complex valued continuous functions, we see that they have a special structure, which is so evident that we usually forget about it: functions form an involutive algebra. Just to recall the obvious - an algebra is a linear space (here taken 
over $\mathbb{C}$ ) equipped with an associative product and an antilinear involution compatible with the product. Rephrasing it in easy words, we may multiply the continuous functions and conjugate them, and the result is a continuous functions. This is, in itself, not so exciting, as we can multiply and conjugate all possible functions, not only the continuous ones. What makes this algebra really interesting is the following:

Remark 3.1. If $X$ is a (locally) compact Hausdorff space and $C(X)$ is the algebra of continuous functions on $X$, then $C(X)$ is a commutative (non) unital $C^{*}$-algebra.

What is a $C^{*}$-algebra? Shortly speaking it is an involutive Banach algebra $A$, that is, a complex normed algebra, which is complete as a topological space in the norm, and for every element $a \in A$ :

$$
\begin{aligned}
& \left\|a a^{*}\right\|=\|a\|^{2} . \\
& \text { 3.1. Examples }
\end{aligned}
$$

We have just met one but a vast set of examples of $C^{*}$-algebra: continuous functions over a (locally) compact topological space. This is, however, not the only one, in particular there are many other $C^{*}$-algebras. Let us see more of them.

Example 3.2 (Finite dimensional $C^{*}$-algebras). For any $n_{1}, \ldots, n_{k}>0$

$$
M_{n_{1}}(\mathbb{C}) \oplus M_{n_{2}}(\mathbb{C}) \oplus \cdots \oplus M_{n_{k}}(\mathbb{C}),
$$

is a $C^{*}$-algebra. In fact, every finite dimensional (as a vector space) $C^{*}$-algebra is of that form.

Before we give two further examples, let us recall that a natural source of algebras comes from linear transformations of a vector space. To have a suitable norm over that algebra, we can take a Hilbert space (which could be finite or infinite) and consider the norm of the linear operator $T$ :

$$
\|T\|=\sup _{v \in \mathcal{H},\|v\|=1}\|T v\| .
$$

All operators for which the norm is defined form an algebra $B(\mathcal{H})$ and are called bounded operators.

Example 3.3 (Bounded operators on $\mathcal{H}$ ). Take a separable Hilbert space $\mathcal{H}$ and $B(\mathcal{H})$, the algebra of all bounded operators on $\mathcal{H}$ (with the operator norm). It is a $C^{*}$-algebra and every norm closed subalgebra of $B(\mathcal{H})$ is a $C^{*}$-algebra. 
Example 3.4 (Compact operators on $\mathcal{H}$ ). The algebra $\mathcal{K}(\mathcal{H})$, which is the closure of the algebra of operators of finite rank in the operator norm is a $C^{*}$-algebra.

Observe that if the Hilbert space is finite dimensional $\mathcal{H}=\mathbb{C}^{n}$ then both the algebra of bounded and compact operators coincide and are isomorphic to $M_{n}(\mathbb{C})$. Finally, a big class of examples comes from groups.

Example 3.5 (Group algebras and Lie groups). For any discrete group $\Gamma$, we can consider the group algebra $\mathbb{C}[\Gamma]$, for any Lie group $G$, we have the convolution algebra over the space of continuous functions with compact support $C_{c}(G)$. Considering various representations of these algebras on Hilbert spaces (and consequently various $C^{*}$-norms), we obtain nontrivial $C^{*}$-algebras related to the respective groups.

\subsection{The Gelfand-Naimark theorems}

So far, we have encountered two types of $C^{*}$-algebra: continuous functions over a particular topological space and algebras of operators on a Hilbert space. We need to learn two important theorems, which set up the scene for further considerations.

Theorem 3.6 (Gelfand-Naimark-Segal [23]). Every abstract $C^{*}$-algebra $\mathcal{A}$ is isometrically $*$-isomorphic to a concrete $C^{*}$-algebra of operators on a Hilbert space $\mathcal{H}$. If the algebra $\mathcal{A}$ is separable then we can take $\mathcal{H}$ to be separable.

Theorem 3.7 (Gelfand-Naimark [22]). If a $C^{*}$-algebra is commutative then it is an algebra of continuous functions on some (locally compact, Hausdorff) topological space.

The lesson drawn from the facts stated above is very simple. First of all, every $C^{*}$-algebra is a norm-closed subalgebra of $B(\mathcal{H})$ for some Hilbert space $\mathcal{H}$. Therefore, we do not need to look at abstract constructions the only thing we need is to look at bounded operators on a Hilbert space. Furthermore, if the algebra is commutative then it is necessarily an algebra of continuous functions on some topological Hausdorff space.

\subsection{More examples of $C^{*}$-algebras}

So far, we had just a couple of examples of $C^{*}$-algebras which were either "classical" (that is described as functions on a space) or rather boring (matrix algebras, all bounded and all compact operators). To see that there are some nontrivial and interesting ones, let us show some easy yet amazing constructions. 
Example 3.8 (Algebra generated by an operator). Let $T$ be a bounded operator on a Hilbert space $\mathcal{H}$ and $T^{*}$ its Hermitian conjugate - we consider the algebra $C^{*}(T)$ as the closure of the algebra of polynomials in $T, T^{*}$.

In particular, if $\mathcal{H}=l^{2}(\mathbb{Z})$ and $T$ is the shift operator $\left(T e_{n}=e_{n+1}\right.$, for the orthonormal basis $\left\{e_{n}\right\}_{n \in \mathbb{Z}}$ of $l^{2}(\mathbb{Z})$ ) then the algebra $C^{*}(T)$ is isomorphic to $C\left(S^{1}\right)$ (all continuous functions on a circle).

Example 3.9 (Toeplitz algebra). Take $\mathcal{H}=l^{2}(\mathbb{N})$ and $T_{s}$ to be a unilateral shift $T_{s} e_{n}=e_{n+1}, n \geq 0$. Then the algebra $C^{*}\left(T_{s}\right)$ is isomorphic to the extension of the algebra $C\left(S^{1}\right)$ by compact operators $\mathcal{K}$, in the following sense, the sequence below is an exact sequence of $C^{*}$-algebras (the image of $i$ is the kernel of $\pi, i$ is injective and $\pi$ is surjective):

$$
0 \rightarrow \mathcal{K} \stackrel{i}{\rightarrow} C^{*}\left(T_{s}\right) \stackrel{\pi}{\rightarrow} C\left(S^{1}\right) \rightarrow 0 .
$$

Example 3.10 (Irrational Rotation Algebra aka Noncommutative Torus). Consider the Hilbert space $L^{2}\left(S^{1}\right)$ and the following operators:

$$
(U f)(z)=z f(z), \quad(V f)(z)=f\left(e^{2 \pi i \theta} z\right)
$$

where $0<\theta<1$ is an irrational real number. We define $\mathbb{T}_{\theta}^{2}$ as a $C^{*}$-algebra generated by the unitary operators $U, V, U^{*}, V^{*}$. We easily check that

$$
U V=e^{2 \pi i \theta} V U \text {. }
$$

\subsection{The first dictionary}

In the previous sections, we have shown an equivalence between commutative $C^{*}$-algebras and spaces. A natural question is whether this analogy could be promoted to other topological constructions, like continuous maps between spaces, Cartesian products etc. The answer is generally yes, and we can write a short list of corresponding operations:

\begin{tabular}{l|l}
\hline \hline \multicolumn{1}{c|}{ Topology } & \multicolumn{1}{c}{ Algebra } \\
\hline locally compact Hausdorff topological space & nonunital $C^{*}$-algebra \\
homeomorphism & automorphism \\
continuous proper map & morphism \\
compact Hausdorff topological space & unital $C^{*}$-algebra \\
open (dense) subset & (essential) ideal \\
compactification & unitization \\
Stone-Čech compactification & multiplier algebra \\
Cartesian product & tensor product
\end{tabular}


Note that, so far, we have just a correspondence between a very rough notion of geometry, which basically tells us about the topology and does not distinguish between a ball and a cube. Of course, there is much more to geometry than topology alone. Noncommutative geometry aims to provide a meaning to the geometric extension of the above table.

\section{How to differentiate?}

In the course of basic analysis, one first defines a derivative of a function, followed by defining the space of differentiable and (finally) smooth functions. A remarkable fact is that thanks to the Leibniz rule both differentiable functions as well the smooth functions are still an algebra.

From the point of view of algebraic approach, the derivative is a derivation of an algebra: a linear map, which obeys the Leibniz rule. If we pass to the noncommutative algebras, we find that derivations split into the trivial ones (those that come from commutators with an element of an algebra such derivations are call inner) and the interesting outer derivations (simply those that are not inner). All derivations of commutative algebras are, of course, outer, whereas for the noncommutative ones, we might have plenty (too many) inner ones and very few (or even none) outer derivations.

Therefore, the idea to repeat the classical construction of derivatives, differentiation and then vector fields (if we pass to the case of manifolds) appears not to be very practical. Instead, we might pass directly to the construction, which in the classical differential geometry is obtained only after some efforts. Here, we need to start with that from the very beginning - with the differential forms.

Let us recall the notion of a module. If $\mathcal{A}$ is an algebra, then we say that $\mathcal{N}$ is a left module, if it is a linear space with a bilinear map: $\mathcal{A} \times \mathcal{N} \ni$ $(a, n) \rightarrow a \cdot n \in \mathcal{N}$ such that:

$$
a \cdot(b \cdot n)=(a b) \cdot n, \quad \forall a, b \in \mathcal{A}, n \in \mathcal{N} .
$$

Similarly, we define a right-module, than we say that $\mathcal{M}$ is a bimodule if it is simultaneously a left and a right module with mutually commuting left and right multiplications:

$$
a \cdot(m \cdot b)=(a \cdot m) \cdot b, \quad \forall a, b \in \mathcal{A}, m \in \mathcal{M} .
$$

Remark 4.1. Differential one-forms $\Omega^{1}(M)$ over a manifold $M$ are a bimodule over the algebra of smooth functions $C^{\infty}(M)$ and the external derivative $d: C^{\infty}(M) \rightarrow \Omega^{1}(M)$ is a bimodule-valued derivation on the algebra $\mathbb{C}^{\infty}(M)$ :

$$
d(f g)=(d f) \cdot g+f \cdot(d g), \quad \forall f, g \in C^{\infty}(M) .
$$


Observe that the external derivative satisfies the Leibniz rule (so it is a derivation) independently of the fact that the algebra is commutative. Therefore, we can define such a bimodule-valued derivation over any algebra, even if it is noncommutative.

Definition 4.2. Let $\mathcal{A}$ be an algebra. The first-order differential calculus $(F O D C)$ over $\mathcal{A}$ is a pair $\left(\Omega^{1}(\mathcal{A}), d\right)$ where $\Omega^{1}(\mathcal{A})$ is a bimodule over $\mathcal{A}$ and $d$ is an $\Omega^{1}(\mathcal{A})$-valued derivation of $\mathcal{A}$ :

$$
d(a b)=(d a) \cdot b+a \cdot(d b), \quad \forall a, b \in \mathcal{A} .
$$

Remark 4.3. Although we can define differential forms and external derivatives for arbitrary (even noncommutative) algebras this definition has a huge drawback: the construction is by no means unique: there are many (even too many) differential calculi over algebras.

To see how it works, let us show some examples.

Example 4.4 (The universal calculus). Let $\mathcal{A}$ be a unital algebra and $\Omega_{u}^{1}(\mathcal{A})=$ ker $\mu$, where

$$
\mu: \mathcal{A} \otimes \mathcal{A} \rightarrow \mathcal{A}, \quad \mu\left(a \otimes a^{\prime}\right)=a a^{\prime} .
$$

The universal FODC is $\Omega_{u}^{1}(\mathcal{A})$ with the universal differential

$$
d_{u}(a)=a \otimes 1-1 \otimes a .
$$

Example 4.5 (Inner FODC). We say that FODC is inner if there exists an element $X \in \Omega^{1}(\mathcal{A})$ such that

$$
d a=X \cdot a-a \cdot X, \quad \forall a \in \mathcal{A} .
$$

Example 4.6 (Two points space and its differential calculus). The algebra of complex-valued functions over a two points space could be identified with the algebra of complex diagonal matrices inside $M_{2}(\mathbb{C})$. We define $\Omega^{1}$ to be all $M_{2}(\mathbb{C})$ matrices and set

$$
d\left(\begin{array}{ll}
c_{1} & 0 \\
0 & c_{2}
\end{array}\right)=\left[\left(\begin{array}{ll}
0 & 1 \\
1 & 0
\end{array}\right),\left(\begin{array}{ll}
c_{1} & 0 \\
0 & c_{2}
\end{array}\right)\right] .
$$

Next, let us compute the universal calculus. An arbitrary element of the algebra is of the form $c_{1} e_{1}+c_{2} e_{2}$, where $c_{1}, c_{2} \in \mathbb{C}, e_{i}^{2}=e_{i}, e_{1} e_{2}=e_{2} e_{1}=$ $0, e_{1}+e_{2}=1$. First,

$$
d e_{i}=e_{i} \otimes 1-1 \otimes e_{i}=e_{i} \otimes e_{i^{\prime}}-e_{i^{\prime}} \otimes e_{i},
$$


where $e_{1^{\prime}}=e_{2}$ and $e_{2^{\prime}}=e_{1}$. Then,

$$
\begin{aligned}
d\left(c_{1} e_{1}+c_{2} e_{2}\right) & =c_{1}\left(e_{1} \otimes e_{2}-e_{2} \otimes e_{1}\right)+c_{2}\left(e_{2} \otimes e_{1}-e_{1} \otimes e_{2}\right) \\
& =\left(c_{1}-c_{2}\right)\left(e_{1} \otimes e_{2}\right)+\left(c_{2}-c_{1}\right)\left(e_{2} \otimes e_{1}\right) .
\end{aligned}
$$

We leave as an easy and entertaining exercise to the Reader to show that the universal calculus is exactly the one as defined earlier using matrices and to check whether this calculus is inner.

Having defined the external derivative, we might want to come a step back and see what happened to the vector fields. In fact, they are not entirely lost - if we consider them not to be acting on the algebra of functions but rather on the Hilbert space on which the algebra is represented faithfully.

Remark 4.7. Imagine the full graded differential algebra over $C^{\infty}(M)$ and take as the Hilbert space the completion (in the $L^{2}$ norm) of the module of one-forms. Then the external derivative $d$ could be seen as densely defined operator on the Hilbert space, and

$$
(d f) \Psi=d(f \cdot \Psi)-f \cdot(d \Psi)=[d, f] \Psi
$$

for $f \in C^{\infty}(M)$ and $\Psi \in \Omega^{*}(M)$.

Therefore, we can encode the derivatives and differential forms through commutators with some operators, which in this case are first-order differential operators. Our aim will be to extend this notion to the general situation of noncommutative algebras.

\section{The Dirac operator}

The external derivative is a natural candidate for a reasonable first-order differential operator, however, as it is certainly not a selfadjoint operator, one must look for its natural extension, the signature operator. This operator falls into the class of generalised Dirac operators.

The recipe to construct the Dirac operator over a spin manifold is quite easy. You start with a Riemannian manifold (compact, closed) with a fixed metric $g$. Find the Clifford algebra bundle, identify the spinor bundle, then lift the metric connection (the Levi-Civita one if you want the true Dirac), then lift it to the spinor bundle, compose with the Clifford map and you get $D$ : the first-order differential operator on the sections of the spinor bundle. Then comes the hard stuff - you need prove some theorems about $D$.

However, we might take a different approach and follow the operational definition, which is as follows. Take an algebra of functions $C^{\infty}(M)$ represented on a Hilbert space of some sections of a suitable vector bundle over $M$ and look for operators, which behave like the Dirac operator. That is, they 
need to be first-order differential operator, from which you can recover the bimodule of differential forms by setting

$$
d a:=[D, a]
$$

as an operator on the Hilbert space. Moreover, from the following formula, you are expected to recover the metric on your manifold

$$
d(x, y)=\sup _{\|[D, f]\| \leq 1, f \in C^{\infty}(M)}|f(x)-f(y)|, \quad \forall x, y \in M .
$$

Additionally, the operator should be unbounded, have discrete spectrum and the eigenvalues should have certain growth property.

In fact, the Dirac operator (and that includes the signature operator as well) carries a lot of information about the additional structures on the manifold. We have already mentioned the differential calculus and the metric. What is also interesting that having the Dirac operator we may directly recover the dimension of the manifold as well as perform the integration using the spectral properties of the Dirac.

\subsection{An interlude: what are vector bundles?}

Before we proceed with the construction of noncommutative Dirac operators, we need to understand what replaces vector bundles when we pass from commutative algebras to noncommutative ones. Let us quote the fundamental theorem.

Theorem 5.1 (Serre-Swan). Let $M$ be a compact manifold, and $E \rightarrow M$ a finite dimensional complex vector bundle. Then the space of continuous sections of $E$ is a finitely generated projective modules over $C(X)$ and every such module is a space of sections of a vector bundle over $M$.

To explain, we need to explain what are projective modules. First, we define a free module over an algebra $\mathcal{A}$, which is just $\mathcal{A}^{n}$ for some $n \geq 1$, with the natural addition and multiplication by the elements of $\mathcal{A}$. Then, we have

Definition 5.2. A module $\mathcal{M}$ over an algebra $\mathcal{A}$ is projective if and only if $\mathcal{M}$ is a summand of a free module, there exists a module $\mathcal{N}$ such that: $\mathcal{M} \oplus \mathcal{N}=\mathcal{A}^{n}$, for some $n \geq 1$.

Let us explain the word "projective" in projective modules, which also demonstrates how to construct projective modules. Take an algebra $\mathcal{A}$ and take a free module $\mathcal{A}^{n}$, then take $p \in M_{n}(\mathcal{A})$ such that $p^{2}=p=p^{*}$. Such $p$ is called a projection, for instance, if $\mathcal{A}=C(M)$ then $p$ is a matrix-valued 
continuous function over $M$ such that $p(x)$ is a projection (as a complex matrix) for every $x \in M$. If we define

$$
\mathcal{M}_{p}=\mathcal{A}^{n} p
$$

then $\mathcal{M}_{p}$ is a projective module. It is easy to see why: if $p$ is a projection so is $1-p$ (so that $p$ and $1-p$ are mutually orthogonal pair of projections) and $\mathcal{M}_{1-p}=\mathcal{A}^{n}(1-p)$ is the complement of $\mathcal{M}_{p}$ so that $\mathcal{M}_{p} \oplus \mathcal{M}_{1-p}=\mathcal{A}^{n}$.

The above construction gives us a finitely generated projective module, so a projective module, which has a finite number of elements which generate it (over the algebra $\mathcal{A}$ ) as a module.

Finally, let us mention one simple though a very interesting property of projective modules: the existence of connections.

Definition 5.3. Let $\mathcal{M}$ be a left module over $\mathcal{A}$ and $\Omega^{1}(\mathcal{A})$ be the firstorder differential calculus over $\mathcal{A}$. The map $\nabla: \mathcal{M} \rightarrow \Omega^{1}(\mathcal{A}) \otimes_{\mathcal{A}} \mathcal{M}$ is a connection if:

$$
\nabla(a \xi)=a \nabla(\xi)+d a \otimes_{\mathcal{A}} \xi, \quad \forall a \in \mathcal{A}, \xi \in \mathcal{M}
$$

Then,

Remark 5.4. If the module $\mathcal{M}$ is projective then there always exists a connection - and, in fact, every module for which connection exists must be projective. This is the seminal result of Cuntz and Quillen [16].

\section{Spectral triples}

We are ready now to put forward a proposition for a generalisation of the Riemannian spin geometry to the realm of noncommutative algebras, which is based on the properties of Dirac operators and constructions we discussed earlier.

Definition 6.1. A spectral triple is a collection of the following data: an algebra $\mathcal{A}$ (which is a dense subalgebra of a $C^{*}$-algebra) together with its faithful representation $\pi$ on a Hilbert space $\mathcal{H}$ and a densely defined selfadjoint operator $D$ (called later Dirac operator), satisfying several conditions:

1. $\forall a \in \mathcal{A}[D, \pi(a)] \in B(\mathcal{H})$,

2. even spectral triples: $\exists \gamma \in \mathcal{A}^{\prime}: \gamma^{2}=1, \gamma=\gamma^{\dagger}, \gamma D+D \gamma=0$,

3. $\exists J$, antilinear $J^{2}= \pm 1, J J^{\dagger}=1$, $J \gamma= \pm \gamma J, J D= \pm D J,[J \pi(a) J, \pi(b)]=0$,

4. $[[D, a], J \pi(b) J]=0$ (D: first-order differential operator), 
5. D has a compact resolvent (so the spectrum of $|D|$ is discrete and its eigenvalues have no other accumulation point than $+\infty$ ),

6. + several conditions (more of "analysis" type), which guarantee that we indeed work with differentiable "functions" and the "noncommutative space" is not singular. We skip details here.

Theorem 6.2. If $\mathcal{A}=C^{\infty}(M), M$ is a spin Riemannian compact manifold, $\mathcal{H}=L^{2}(S)$ is the Hilbert space of summable sections of the spinor bundle and $D$ is the Dirac operator on $M$ then to $(\mathcal{A}, \mathcal{H}, D)$ is a spectral triple (with a real structure).

The definition (more or less in this form) was proposed by Connes in [10] then developed later by many authors. Details of the proof of the above theorem could be found in [2].

\subsection{Examples of spectral triples}

Apart from the classical case of smooth functions over a compact spin manifold, there are several examples of genuinely noncommutative spectral geometries. Before we list them, let us mention that spectral triples over commutative algebras (which satisfy some additional requirements) are in fact only of that type, thanks to Connes' reconstruction theorem [14]. Hence, commutative spectral triples are equivalent (in the sense of 1:1 correspondence) to compact spin manifolds.

Thus we might look for the noncommutative examples as some nontrivial generalisations of spin manifolds to (using a figure of speech) "noncommutative manifolds".

Let us list some of the examples with the relevant references:

- The Noncommutative Torus: $U V=e^{2 \pi i \theta} V U$

Dirac operator the same as on the torus [10,35].

- Theta deformations of manifolds (NC Torus is a special case) Dirac operator the same as on the original manifold [15].

- Finite matrix algebras: $M_{n_{1}}(\mathbb{C}) \oplus M_{n_{2}}(\mathbb{C}) \oplus \cdots$ Dirac operator is a finite Hermitian matrix [30, 33].

- Quantum spaces (q-deformations of spheres, $\mathrm{SU}_{q}(2)$ ) Requires some modifications of the definition [17, 18].

- Moyal deformation $\left[x^{\mu}, x^{\nu}\right]=\theta^{\mu \nu}$ The same Dirac as on $\mathbb{R}^{2 n}$ [21]. 
- $\kappa$-deformation $\left[x^{0}, x^{i}\right]=\frac{1}{i \kappa} x^{i}$, where $\kappa$ is a real parameter, relevant for Doubly Special Relativity, requires twisting of spectral triples [26, 31] .

Remark 6.3 (How to construct spectral triples?). There is so far no general method. Mathematically, spectral triple is an unbounded Fredholm module, a representative of the $K$-homology class of the algebra $\mathcal{A}$, hence taking a representative of that class gives a bare skeleton to construct a spectral triple. However, there are very few general results whether this is possible, see [39] for details.

\subsection{Getting some numbers}

In physics, having a general construction of the model is just the starting point, which needs to be later developed to a theory, in which, at the end, we want to obtain some numbers. This is, in fact, what is hidden all over in physics: computing the action in the classical mechanics and classical field theory and calculating expectation values in quantum mechanics and quantum field theory.

Therefore, we need to learn how to use the tools defined above to get down to things, which are indeed computable. To reach this aim, we need to use some of the advanced theory of exotic traces, using the methods, which resemble the regularisation techniques.

\subsection{Regularisation and exotic traces}

Imagine we have a positive operator $T$ on an infinite dimensional separable Hilbert space, such that its spectrum is discrete. Then, arranging its eigenvalues in an increasing order (and taking into account the multiplicities), we might define

$$
\operatorname{Tr}_{\omega}(T)=\lim _{N \rightarrow \infty} \frac{1}{\log N} \sum_{i=1}^{N} \mu_{i}(T),
$$

where $\mu_{i}(T)$ is the $i$ th eigenvalue of $T$.

Of course, such limit may not even exist and certainly it does not for a huge class of operators, whereas for some it is just $+\infty$. However, there exists a big family of operators, for which the limit exists and is finite and, moreover, that family is an ideal in the algebra of bounded operators on that Hilbert space. What is even more surprising, this functional is then a trace, so

$$
\operatorname{Tr}_{\omega}(T S)=\operatorname{Tr}_{\omega}(S T)
$$

for $T, S$ such that the functional is well defined on their product. This was found by Dixmier, hence the functional is called Dixmier trace and is an 
example of so-called exotic traces. Note, that if $T$ is trace class itself then, of course, $\operatorname{Tr}_{\omega}(T)=0$. For this reason, the Dixmier trace is nothing else but a regularisation of the usual trace for the family of operators, for which the trace is a priori infinite.

One may wonder whether some other methods of regularisations used in quantum field theory, for instance, the zeta function regularisation, may be also applied. Surprisingly, yes and in the same spirit we may define the following functionals.

Let $T$ be a compact positive operator such that for sufficiently large $r>0$ the operator $T^{r}$ is trace class. Therefore, the function

$$
\zeta_{T}(z):=\operatorname{Tr}|T|^{z}
$$

is well defined and holomorphic for $\Re(z)>r$. Taking the analytic continuation of $\zeta_{T}(z)$ to the rest of the complex plane, we obtain a function, which has (possibly) some poles. We may then set for any $d \in \mathbb{R}$

$$
\tau(T):=\operatorname{Res}_{z=d} \zeta_{T}(z) .
$$

It appears that for each $d$ there exists a large class of operators, so that the residue is nontrivial and for some, the functional $\tau$ has again the trace property.

In fact, if $D$ is the Dirac operator on a spin manifold of dimension $n$ then the function $\zeta_{|D|^{-1}}$ (if $D$ has a kernel it is certainly finite dimensional and we can neglect it) may have only first-order poles only at integers on the real axis not exceeding $n$ and, in particular, has a nonzero residue at $z=n$. Just to shorten the notation, we shall write $\zeta_{D}$ (meaning $\zeta_{|D|^{-1}}$ ).

Example 6.4. Take an example of a circle, $S^{1}$. The Hilbert space $L^{2}\left(S^{1}\right)$ has an orthonormal basis $e_{n}, n \in \mathbb{Z}$ and the usual Dirac operator is De $e_{n}=$ $n e_{n}$. The $\zeta$ function of the Dirac operator on the circle is:

$$
\zeta_{D}(z)=2 \sum_{n=1}^{\infty} \frac{1}{n^{z}}=2 \zeta(z) .
$$

Having the zeta function, we may look at its poles which are located generally in a half of the complex plane and are not necessarily real. The collection of all points, which are the poles of the zeta function of the operator $D$ from a given spectral triple we call the dimension spectrum. So, the dimension is not a number - it is a discrete set in a complex plane!

Remark 6.5. The dimension spectrum of a compact spin manifold $M$, given by its spectral triple $\left(C^{\infty}(M), L^{2}(S), D\right)$ is contained in a set: $\{n, n-1$, $n-2, \ldots\}$, where $n$ is the classical dimension of $M$. In fact, $z=n$ is always in the dimension spectrum, whereas not all of other the points of the set may belong to the dimension spectrum. 
Remark 6.6. The dimension spectrum may contain complex numbers (with nonzero imaginary part) and any real numbers (for instance, if one considers fractals).

\section{Towards physics}

Our aim in this section will be to construct a classical field theory, which mimics the gauge theory and formulate it using exclusively with the use of the tools of noncommutative geometry.

First of all, having a "noncommutative geometry" in terms of a spectral triple, we may wonder what is the set of all possible physical degrees of freedom - that is physical fields. Keeping in mind that we consider the geometry - the appropriate choice of fields is like in the case of gravity where the relevant field is the metric. Therefore, since in the case of spectral triples metric is replaced by the Dirac operator, we need to take as a dynamical variable of our theory the Dirac operator itself.

Our initial data is therefore an algebra (a suitable dense subalgebra of a $C^{*}$-algebra), its representation on the Hilbert space (elements of which are to be identified with spinor fields) and the set of fields, which are identified with all possible Dirac operators.

Of course, we need to understand how to find a family of Dirac operators. Classically, on a spin manifold, the Dirac operator depends alone on the metric, however, if we twist the spinor bundle (by tensoring it with an additional vector bundle) and twist the Dirac by a connection on the additional bundle, we still have a Dirac operator. In the noncommutative situation, this translates to the so-called inner fluctuations.

Remark 7.1 (Family of Dirac operators). Once we have Dirac operator for a given spectral triple, we have an entire family of them by taking all inner fluctuations of Dirac operators:

$$
\mathrm{D}_{A}=\left\{D^{\prime}: D^{\prime}=D+A\right\},
$$

where $A$ is a self-adjoint one-form $A=\sum_{i} a_{i}\left[D, b_{i}\right]$. Classically, this corresponds to the twisting of the Dirac operator by a (trivial) complex line bundle, or - using physics terminology - so adding U(1) gauge field.

Of course, one could ask a question whether the family we get depends on the starting point (that is whether the family is the same if we start with the Dirac already perturbed by a one-form) and it is very convenient that indeed the inner fluctuation of inner fluctuation are inner fluctuations so the family we obtain is not dependent on the initial choice. 
Remark 7.2 (A warning). The space of all possible Dirac operators is much bigger in the classical case than the space of metrics (but for real spectral geometries it excludes U(1) gauge connections). The Dirac operators with torsion are, for instance, allowed.

\subsection{A proposition for the action ...}

Having fixed the background for the physical model (geometry and space of dynamical fields), we can look for the action - the universal principle in all of fundamental theoretical physics. Recall, that our physical field is, in fact, not a field but an operator $D$, which belongs to a family of operators, which we shall denote $D_{g, A}$.

Definition 7.3 (Let the action be spectral!). For a fixed function $f$ (playing the role of cutoff), consider the following functional on the space of $D_{g, A}$

$$
S\left(D_{g, A}\right)=\operatorname{Tr} f\left(\frac{D_{g, A}^{2}}{\Lambda^{2}}\right) .
$$

Note that the above expression makes sense only for certain functions $f$, in particular, the standard choice will be the Heaviside function $f(x)=1$ for $0 \leq x \leq 1$ and 0 otherwise. In such a case, the action just counts the number of eigenvalues of the Dirac operator, which are (their absolute value) less (or equal) $\Lambda$. As the action depends explicitly on the function $f$ and the cutoff parameter $\Lambda$, we might wonder whether one can read out of it anything interesting. The answer comes from the asymptotic expansion, that is the expansion of the action functional as a polynomial in $\Lambda$.

Lemma 7.4. With certain assumptions on the dimension spectrum Sd of the spectral triple (discrete, real points with simple poles) we have that for large $\Lambda$

$$
S\left(D_{g, A}, f, \Lambda\right)=\sum_{k \in S d^{+}} f_{k} \Lambda^{k}\left(\operatorname{Res}_{z=k} \zeta_{D_{g, A}}(z)\right)+f(0) \zeta_{D_{g, A}}(0)+\mathcal{O}\left(\Lambda^{-2}\right),
$$

where $S d^{+}$is the positive part of the dimension spectrum of $(\mathcal{A}, \mathcal{H}, D)$ and the coefficients $f_{k}=\frac{1}{2} \int_{0}^{\infty} f(t) t^{k / 2-1} d t$ are the momenta of the cutoff function $f$. Moreover, the scale invariant part of the spectral action (independent of $\Lambda$ ) depends on the inner perturbation $A$ in the following way:

$$
\zeta_{D_{A}}(0)-\zeta_{D}(0)=\sum_{q=1}^{n} \frac{(-1)^{q}}{q} \operatorname{Res}_{z=d}\left(\operatorname{Tr}\left(\left(A D^{-1}\right)^{q}|D|^{-z}\right)\right) .
$$

The spectral action in its form was proposed by Chamseddine and Connes [5] and developed later in [9] but the idea could be traced back to Sakharov [37]. 


\subsection{The meaning of all that}

Before we explain in details the terms of the spectral action which arise, let us discuss what the spectral action gives if we consider a genuine, true, metric-dependent Dirac operator over a compact spin manifold of a physically relevant dimension $D$.

If $M$ is a compact spin manifold of dimension 4 and $D$ is the Dirac operator over the spinor bundle (for a particular choice of a spin structure) then the spectral action has the following expansion:

$$
\begin{aligned}
S(D)= & \frac{1}{2 \pi^{2}} f_{4} \Lambda^{4} \int \sqrt{g} d^{4} x+\frac{1}{24 \pi^{2}} f_{2} \Lambda^{2} \int R \sqrt{g} d^{4} x \\
& -\frac{1}{320 \pi^{2}} f(0) \int C_{\mu \nu \rho \sigma} C^{\mu \nu \rho \sigma} \sqrt{g} d^{4} x+O\left(\Lambda^{-2}\right),
\end{aligned}
$$

where $R$ is the scalar curvature, $C_{\mu \nu \rho \sigma}$ is the Weyl tensor and we omit the topological term (corresponding to Gauss-Bonnet term but in 4 dimensions).

So we have recovered the usual gravity (albeit Euclidean!) in 4-dimensions, featuring the cosmological constant (leading term at $\Lambda^{4}$ ), the EinsteinHilbert action (the term at $\Lambda^{2}$ ) and some scale-invariant corrections, often considered by people investigating extensions of general relativity models.

To go beyond the classical case (since in the above example although we use the techniques of noncommutative geometry the entire construction is still classical), let us merge the model we just studied with the one mentioned earlier, the one that describes the geometry of a two-point space.

Example 7.5 (A spectral triple over two points). Consider the example of the algebra of functions over two points, $A_{2}$ identified with the diagonal matrices in $M_{2}(\mathbb{C})$. We shall give an example of a real spectral triple, which yields the differential calculus described earlier. It appears that to satisfy all conditions of a real spectral triple one cannot take the irreducible representation of $A_{2}$ (which would be on $\mathbb{C}^{2}$ ). The reason for this is simple, the existence of the real structure requires that the Hilbert space becomes a module over differential forms, such that the action of one-forms commutes with the right multiplication by the elements of the algebra. The smallest representation space, which allows is on $\mathbb{C}^{2} \otimes \mathbb{C}^{2}$, where we have

$$
\begin{aligned}
A_{2} \ni a & =\left(\begin{array}{cc}
c_{+} & 0 \\
0 & c_{-}
\end{array}\right) \otimes i d, \\
J(v \otimes w) & =w^{*} \otimes v^{*}, \quad v, w \in \mathbb{C}^{2} .
\end{aligned}
$$

The grading $\gamma$ could be any diagonal matrix on $\mathbb{C}^{2} \otimes \mathbb{C}^{2}$ with entries \pm 1 , and the Dirac operator any selfadjoint linear operator on $\mathbb{C}^{2} \otimes \mathbb{C}^{2}$, which anticommutes with $\gamma$, commutes with $J$ and satisfies the order one condition. 
Using explicitly elements of $M_{4}$, we rewrite first the representation and $J$

$$
\begin{aligned}
& A_{2} \ni a=\left(\begin{array}{llll}
c_{+} & 0 & 0 & 0 \\
0 & c_{+} & 0 & 0 \\
0 & 0 & c_{-} & 0 \\
0 & 0 & 0 & c_{-}
\end{array}\right), \\
& J\left(\begin{array}{c}
v_{1} \\
v_{2} \\
w_{1} \\
w_{2}
\end{array}\right)=\left(\begin{array}{c}
v_{1}^{*} \\
w_{1}^{*} \\
v_{2}^{*} \\
w_{2}^{*}
\end{array}\right)
\end{aligned}
$$

so that $J a^{*} J$ becomes

$$
J a^{*} J=\left(\begin{array}{llll}
c_{+} & 0 & 0 & 0 \\
0 & c_{-} & 0 & 0 \\
0 & 0 & c_{+} & 0 \\
0 & 0 & 0 & c_{-}
\end{array}\right) .
$$

The grading $\gamma$ compatible with $J$, so that $J \gamma=\gamma J$ is

$$
\gamma\left(\begin{array}{c}
v_{1} \\
v_{2} \\
w_{1} \\
w_{2}
\end{array}\right)=\left(\begin{array}{c}
+v_{1} \\
-v_{2} \\
-w_{1} \\
+w_{2}
\end{array}\right) .
$$

The Dirac operator is a priori a selfadjoint matrix in $M_{4}(\mathbb{C})$, however, using its commutation relations with $\gamma, J$ and the order-one condition, one can actually find that the following satisfies all conditions (the Reader is encouraged to check whether this is the only one, and if not to find all such operators)

$$
D\left(\begin{array}{l}
v_{1} \\
v_{2} \\
w_{1} \\
w_{2}
\end{array}\right)=\left(\begin{array}{l}
\phi w_{1}+\phi^{*} v_{2} \\
0 \\
0 \\
\phi^{*} v_{2}+\phi w_{1}
\end{array}\right) .
$$

To see, let us check $D J=J D$

$$
\begin{gathered}
D J\left(\begin{array}{l}
v_{1} \\
v_{2} \\
w_{1} \\
w_{2}
\end{array}\right)=\left(\begin{array}{l}
\phi v_{2}^{*}+\phi^{*} w_{1}^{*} \\
0 \\
0 \\
\phi^{*} w_{1}^{*}+\phi v_{2}^{*}
\end{array}\right), \quad J D\left(\begin{array}{l}
v_{1} \\
v_{2} \\
w_{1} \\
w_{2}
\end{array}\right)=\left(\begin{array}{l}
\phi^{*} w_{1}^{*}+\phi v_{2}^{*} \\
0 \\
0 \\
\phi v_{2}^{*}+\phi^{*} w_{1}^{*}
\end{array}\right), \\
D=\left(\begin{array}{llll}
0 & \phi^{*} & \phi & 0 \\
\phi & 0 & 0 & 0 \\
\phi^{*} & 0 & 0 & 0 \\
0 & 0 & 0 & 0
\end{array}\right)
\end{gathered}
$$


Finally, for the order one condition, we compute

$$
[D, a]=\left(\begin{array}{llll}
0 & 0 & \phi\left(c_{-}-c_{+}\right) & 0 \\
0 & 0 & 0 & 0 \\
\phi^{*}\left(c_{+}-c_{-}\right) & 0 & 0 & 0 \\
0 & 0 & 0 & 0
\end{array}\right)
$$

so that

$$
J[D, a] J=\left(\begin{array}{llll}
0 & \phi^{*}\left(c_{-}-c_{+}\right)^{*} & 0 & 0 \\
\phi\left(c_{+}-c_{-}\right)^{*} & 0 & 0 & 0 \\
0 & 0 & 0 & 0 \\
0 & 0 & 0 & 0
\end{array}\right)
$$

and it is clear that the order-one condition is satisfied.

The details on how to construct finite spectral triples, that is triples over finite dimensional algebras could be found in [33] and [30], the use of symmetries to restrict some Dirac operators to equivariant ones was further developed in [36].

Example 7.6 (The two-sheet spacetime). Consider the algebra of smooth functions over a 4-dimensional compact spin manifold $M$, which are valued in diagonal matrices within $M_{2}(\mathbb{C})$. This algebra is indeed the algebra of functions on two disjoint copies of the same manifold, yet using noncommutative geometry we shall be able to say a lot more about its geometry than the usual differential geometry teaches us. The reason for this is that spectral triples exist for both components: the classical manifold as well as the discrete two-point space and there is an easy method to construct a spectral triple for the product geometry.

Let $\gamma_{M}, J_{M}, D_{M}$ denote the $\mathbb{Z}_{2}$-grading, real structure and the Dirac operator for the manifold and $\gamma_{F}, J_{F}, D_{F}$ denote the same objects for the spectral triple over two-point space we described in the previous example.

Then, by taking the tensor algebra $C^{\infty}(M) \otimes A_{2}$, and its representation on $L^{2}(S) \otimes V$, where $S$ denotes a chosen spinor bundle over $M$ and $V$ is a finite dimensional space on which we represent the finite subalgebra (so, for the two-point space it is $\mathbb{C}^{4}$, and setting

$$
\begin{aligned}
\gamma & =\gamma_{M} \otimes \gamma_{F}, \\
J & =J_{M} \otimes J_{F}, \\
D & =D_{M} \otimes \gamma_{F}+i d \otimes D_{F},
\end{aligned}
$$

we obtain a real spectral triple for the algebra of smooth functions over the product geometry $M \times \mathbb{Z}_{2}$ (two-point space could be identified with the $\mathbb{Z}_{2}$ group). 
Observe that $D^{2}$ is easily calculated

$$
D^{2}=D_{M}^{2}+D_{F}^{2},
$$

and using the results from the previous example (so assuming the form of the Dirac operator we have taken), we obtain

$$
D^{2}=D_{M}^{2} \otimes i d+i d \otimes 2 \phi \phi^{*} E_{1},
$$

where $\phi$ becomes a complex-valued scalar field over $M$, and $E_{1}$ is the rank 1 projection in $M_{4}(\mathbb{C})$.

Finally, we can use that to compute the spectral action, and compare with the previous result for the "pure gravity case". We obtain (again, up to topological terms)

$$
\begin{aligned}
S(D)= & \frac{2}{\pi^{2}} f_{4} \Lambda^{4} \int \sqrt{g} d^{4} x+\frac{1}{6 \pi^{2}} f_{2} \Lambda^{2} \int\left(R+\frac{1}{2} \phi \phi^{*}\right) \sqrt{g} d^{4} x \\
& +\frac{1}{320 \pi^{2}} f(0) \int\left(-4 C_{\mu \nu \rho \sigma} C^{\mu \nu \rho \sigma}+10\left(\phi \phi^{*}\right)^{2}+\frac{10}{3} R\left(\phi \phi^{*}\right)\right) \sqrt{g} d^{4} x \\
& +O\left(\Lambda^{-2}\right) .
\end{aligned}
$$

When we forget about the gravity (thus ignoring all terms with $R$ and the Weyl tensor), we see that we obtain a model for a classical complex field theory with the natural potential that contains a quadratic $\phi \phi^{*}$ and quartic $\left(\phi \phi^{*}\right)^{2}$ terms.

\subsection{A more refined model ...}

In the above model, which was just a toy example whose only purpose was to indicate that playing with some finite geometries one may get nontrivial results, we have ignored all other internal fluctuations of the Dirac and omit some other admissible operators. Generally, for the geometries of the type $M \times F$, where $M$ is a Riemannian manifold and $F$ is a discrete geometry (not necessarily of a commutative type), we shall obtain similar results as in the previous section with the addition of the Yang-Mills action for the usual, gauge-type perturbations of the Dirac operator. 
Let us look at the following action, which strongly resembles something quite well known for particle physicists

$$
\begin{aligned}
S= & \frac{1}{\pi^{2}}\left(48 f_{4} \Lambda^{4}-f_{2} \Lambda^{2} c+\frac{f_{0}}{4} d\right) \int \sqrt{g} d^{4} x \\
& +\frac{96 f_{2} \Lambda^{2}-f_{0} c}{24 \pi^{2}} \int R \sqrt{g} d^{4} x \\
& +\frac{f_{0}}{10 \pi^{2}} \int\left(\frac{11}{6} R^{*} R^{*}-3 C_{\mu \nu \rho \sigma} C^{\mu \nu \rho \sigma}\right) \sqrt{g} d^{4} x \\
& +\frac{\left(-2 a f_{2} \Lambda^{2}+e f_{0}\right)}{\pi^{2}} \int|\varphi|^{2} \sqrt{g} d^{4} x \\
& +\frac{f_{0}}{2 \pi^{2}} \int a\left|D_{\mu} \varphi\right|^{2} \sqrt{g} d^{4} x \\
& -\frac{f_{0}}{12 \pi^{2}} \int a R|\varphi|^{2} \sqrt{g} d^{4} x \\
& +\frac{f_{0}}{2 \pi^{2}} \int\left(g_{3}^{2} G_{\mu \nu}^{i} G^{\mu \nu i}+g_{2}^{2} F_{\mu \nu}^{\alpha} F^{\mu \nu \alpha}+\frac{5}{3} g_{1}^{2} B_{\mu \nu} B^{\mu \nu}\right) \sqrt{g} d^{4} x \\
& +\frac{f_{0}}{2 \pi^{2}} \int b|\varphi|^{4} \sqrt{g} d^{4} x,
\end{aligned}
$$

where $c, b, a, g_{1}, g_{2}, g_{3}$ are some constants, which correspond to the parameters of the models (and that includes the masses of fermions and gauge couplings).

How is it done? First, we choose a suitable finite geometry

$$
F=\mathbb{C} \oplus \mathbb{H} \oplus M_{3}(\mathbb{C}) .
$$

Then, by taking a product geometry

$$
\mathcal{A}=C^{\infty}(M) \otimes F,
$$

where $M$ is a 4-dimensional compact spin manifold and taking a suitable choice of the representation of the algebra, the real structure and the grading on the finite algebra (which could be either guessed, derived from some ad hoc principles or just read from the contents of particle physics textbooks), we can look for all admissible Dirac operators. The first free parameter is obviously the metric on $M$, then the space of all internal fluctuations of the Dirac. The space-like internal fluctuations are one forms valued in some subspace of the algebra. Since the algebra is semisimple, we can split them into three parts and identify the relevant one-forms as arising from a $\mathrm{U}(1), \mathrm{SU}(2)$ and $\mathrm{SU}(3)$ connections (it helps that the unitary elements of the algebra $F$ form a $\mathrm{U}(1) \times \mathrm{SU}(2) \times \mathrm{U}(3)$ group, adding unimodularity condition one recovers the Standard Model gauge connections). 
In the discrete space direction, we have almost the same situation as in the model of two-point space we discussed. The exact form of the reality structure and the admissible Dirac (though one needs some extra ad hoc principle to avoid some leptoquarks, see [34] for details) enforces that the only field, which appears is quaternionic-valued and (since quaternions are in fact a pair of complex numbers) we recover something, which is easily interpreted as the Higgs doublet.

So, on the classical level, we have reinterpreted the Lagrangian of the Standard Model as arising from some Kaluza-Klein type geometry but with the geometry playing the role of compact dimension being genuinely noncommutative (though it is the easiest and very mild type of noncommutativity). By doing that, we did reconstruct all the terms in the classical bosonic Lagrangian (there is no problem with the action for fermions, where one recovers the minimal coupling as in the Standard Model) including the Higgs doublet symmetry-breaking potential. All fields in this model have a geometric interpretation as gauge potentials (they come as the parameters of the admissible Dirac operators) and the action arises in a completely natural way, without any terms inserted "by hand".

Of course, generally, there is much more freedom in the choice of the Dirac operators, than we have discussed here. There is a possibility of torsion or other perturbations [24, 40], dilaton field and some additional fermionic terms if one includes extra fermions and looks for Majorana mass terms [6]. Nevertheless, even if the story is not finished and the picture has many gaps, it becomes obvious that the Standard Model as it is, with the Higgs and the symmetry breaking mechanism has a beautiful geometric interpretation.

To end this section, let us note that there are several ways to choose the algebra and the Hilbert space. For instance, one can start from a bigger algebra (see [13] for details), take its fundamental representation and then look for all possible Dirac operators, which satisfy order one connection and connect all disconnected components. Take $\mathcal{A}_{F}$ as the maximal subalgebra such that $\left[D, \mathcal{A}_{F}\right] \neq 0$. Equivalently, one may just take the input from physics or look for some symmetries (including the natural idea of the covering of rotation group: its double cover gives spinors and though no nontrivial covers are possible in the groups, there exist a nontrivial cover in the noncommutative world, in the category of Hopf algebras). All these ideas require further exploration. 


\section{Conclusions}

Having started this review with some dictionary reviewing the topological counterparts in the language of spaces and algebras, we might propose its extension. It should not be treated as a definitive one but just a hint that geometry is much more extensive and bigger than we might have originally believed. Many of the notions, which are listed below, have not even been mentioned here - we refer the Reader to many textbooks and review papers, which we list later.

\subsection{The dictionary (continued)}

\begin{tabular}{l|l}
\hline \hline \multicolumn{1}{c|}{ Geometry } & \multicolumn{1}{c}{ Algebra } \\
\hline sections of a vector bundle & $\begin{array}{l}\text { finitely generated projective module } \\
\text { differential forms }\end{array}$ \\
differential forms & Hochschild homology \\
de Rham cohomology & cyclic cohomology \\
vector fields & operators \\
group & Hopf algebra \\
Lie algebra & Hopf algebra \\
principal fibre bundle & Hopf-Galois extension \\
measurable functions & von Neumann algebra \\
infinitesimals & compact operators \\
metric & Dirac operator \\
spinc geometry & spectral triple \\
spin geometry & real spectral triple \\
integrals & exotic traces
\end{tabular}

To summarise, let us say that noncommutative geometry is a tool, which allows to describe geometry more generally and allows for much broader scope than differential geometry alone. Now, there is a way to study geometry (in such a language) of discrete spaces, fractals, noncommutative algebras - and it is all sound and beautiful mathematics.

Of course, one should distinguish between the methods (shown here), the models applicable to particle physics (like the one discussed above) and some toy models that aim to study "quantum spacetime" (like Moyal deformation or the $\kappa$-Minkowski). Those models are, however, a very useful tool in our way towards understanding the structure and the geometry of spacetime.

\subsection{Further reading}

The mathematical foundations of noncommutative geometry could be found in the original paper by Connes [7] and the book [8]. More recent book by Connes and Marcolli [13] includes also details on the noncommutative 
description of the Standard Model. A good basic and thorough textbook (again, mathematically oriented) is the one by Gracia-Bondia, Várilly and Figureoa [2], some other aspects are presented in a textbook by Khalkhali [29]. There are numerous old and new reviews and lecture notes like [11, 12], a collection of short expositions [38] and some expository articles [28]. A slightly different approach could be found in [20]. Specific explanation on the Standard Model (more on to the physics side) could be found in [27], a similar (more mathematical review) in [41]. A concise and excellent review of spectral geometry, spectral action and exotic traces is in [25].

\section{REFERENCES}

[1] Atlas Collaboration, Phys. Lett. B716, 1 (2012).

[2] J.M. Bondia, J. Várilly, H. Figueroa, Elements of Noncommutative Geometry, Birkhauser Advanced Texts, Birkhauser Boston, Inc., Boston, MA, 2001.

[3] S. Capozziello, G. Lambiase, New Adv. Phys. 7, (2013).

[4] A.H. Chamseddine, A. Connes, Phys. Rev. Lett. 77, 4868 (1996).

[5] A.H. Chamseddine, A. Connes, Commun. Math. Phys. 186, 731 (1997).

[6] A. Chamseddine, A. Connes, M. Marcolli, Adv. Theor. Math. Phys. 11, 991 (2007).

[7] A. Connes, Publ. Math. Inst. Hautes Études Sci. 41, 257 (1985).

[8] A. Connes, Noncommutative Geometry, Academic Press, Inc, San Diego 1994.

[9] A. Connes, Commun. Math. Phys. 117, 673 (1988).

[10] A. Connes, J. Math. Phys. 36, 6194 (1995).

[11] A. Connes, J. Math. Phys. 41, 3832 (2000).

[12] A. Connes, in: Visions in Mathematics, GAFA 2000 Special Volume, Part II, (Eds.) N. Alon, J. Bourgain, A. Connes, M. Gromov, V. Milman, Modern Birkhäuser Classics, 2010, pp. 481-559.

[13] A. Connes, M. Marcolli, Noncommutative Geometry, Quantum Fields and Motives, American Mathematical Society, Providence, RI; Hindustan Book Agency, New Delhi 2008.

[14] A. Connes, J. Nonc. Geom. 7, 1 (2013).

[15] A. Connes, G. Landi, Commun. Math. Phys. 221, 141 (2001).

[16] J. Cuntz, D. Quillen, J. Amer. Math. Soc. 8, 251 (1995).

[17] L. Dabrowski et al., Commun. Math. Phys. 259, 729 (2005).

[18] L. Dabrowski, A. Sitarz, Banach Center Publ. 61, 49 (2003).

[19] S. Doplicher, K. Fredenhagen, J. Roberts, Commun. Math. Phys. 172, 187 (1995).

[20] M. Dubois-Violette, Contemp. Math. 294, 59 (2002). 
[21] V. Gayral et al., Commun. Math. Phys. 246, 569 (2004).

[22] I.M. Gelfand, M.A. Naimark, Math. Sbornik 12, 197 (1943).

[23] I.E. Segal, Bull. Am. Math. Soc. 53, 73 (1947).

[24] F. Hanisch, F. Pfäffle, Ch. Stephan, Commun. Math. Phys. 300, 877 (2010).

[25] B. Iochum, "Spectral Geometry", Lecture Notes from "Geometric, Algebraic and Topological Methods for Quantum Field Theory, 2011, Villa de Leyva, Columbia, 2011.

[26] B. Iochum, T. Masson, T. Schücker, A. Sitarz, Rep. Math. Phys. 68, 37 (2011).

[27] J-H. Jureit, T. Krajewski, T. Schücker, Ch. Stephan, Acta Phys. Pol. B 38, 3181 (2007).

[28] D. Kastler, J. Math. Phys. 41, 3867 (2000).

[29] M. Khalkhali, Basic Noncommutative Geometry, EMS Series of Lectures in Mathematics, 2009.

[30] T. Krajewski, J. Geom. Phys. 28, 1 (1998).

[31] M. Matassa, J. Geom. Phys. 76, 136 (2014) [arXiv:1212.3462 [math-ph]].

[32] S. Neshveyev, L. Tuset, Journal für die Reine und Angewandte Mathematik 641, 1 (2010).

[33] M. Paschke, A. Sitarz, J. Math. Phys. 39, 6191 (1998).

[34] M. Paschke, F. Scheck, A. Sitarz, Phys. Rev. D59, 035003 (1999).

[35] M. Paschke, A. Sitarz, Lett. Math. Phys. 77, 317 (2006).

[36] M. Paschke, A. Sitarz, Acta Phys. Pol. B 31, 1897 (2000).

[37] A. Sakharov, Dokl. Akad. Nauk Ser. Fiz. 177, 7 (1967).

[38] F. Scheck et al., Lect. Notes Phys. 596, 230 (2002).

[39] E. Schrohe, M. Walze, J.-M. Warzecha, C. R. Acad. Sci. Paris 326, 1195 (1998).

[40] A. Sitarz, A. Zajac, Lett. Math. Phys. 98, 333 (2011).

[41] A. Sitarz, Acta Polytechnica 48, 34 (2008). 